\title{
EXPLORING THE NATURE OF COMPACT RADIO SOURCES ASSOCIATED TO UCHII REGIONS
}

\author{
Josep M. Masqué ${ }^{1}$, Luis F. Rodríguez ${ }^{2,3}$, Sergio A. Dzib ${ }^{4}$, S.-N. X. Medina ${ }^{4}$, Laurent Loinard ${ }^{2}$, \\ Miguel A. Trinidad ${ }^{1}$, Stan Kurtz ${ }^{2}$, and Carlos A. Rodríguez-Rico ${ }^{1}$ \\ Received August 25 2020; accepted October 72020
}

\begin{abstract}
We present Very Large Array $7 \mathrm{~mm}$ continuum observations of four ultracompact (UC) HII regions, observed previously at $1.3 \mathrm{~cm}$, in order to investigate the nature of the compact radio sources associated with these regions. We detect a total of seven compact radio sources, four of them with thermal emission, and two compact radio sources with clear non-thermal emission. The thermal emission is consistent with the presence of an ionized envelope, either static (i.e., trapped in the gravitational radius of an associated massive star) or flowing away (i.e., a photo-evaporative flow). The nature of the non-thermal sources remains unclear and several possibilities are proposed. The possibility that most of these compact radio sources are photo-evaporating objects, and the remaining ones more evolved objects, is consistent with previous studies on UCHII regions.
\end{abstract}

\section{RESUMEN}

Presentamos observaciones del continuo a $7 \mathrm{~mm}$ con el Very Large Array de cuatro regiones HII ultra-compactas (UCHII), observadas previamente a $1.3 \mathrm{~cm}$, con el fin de investigar la naturaleza de las fuentes de radio compactas asociadas con estas regiones. Detectamos un total de siete radio fuentes compactas, cuatro de ellas con emisión térmica, y dos radio fuentes compactas claramente con emisión no térmica. La emisión térmica es congruente con la presencia de una envolvente ionizada estática (i.e., atrapada en el pozo de potencial de la estrella masiva asociada a la región) o fluyendo (i.e., un flujo foto evaporándose). La naturaleza de las fuentes no térmicas no es clara y se proponen algunas posibilidades. La posibilidad de que la mayoría de estas fuentes de radio compactas sean objetos foto evaporándose y las fuentes restantes objetos más evolucionados concuerda con estudios previos sobre regiones UCHII.

Key Words: HII regions - stars: formation

\section{INTRODUCTION}

The advent of a new generation of upgraded instruments (e.g. the Karl G. Jansky Very Large Array) enables radio astronomers to detect and systematically study the weakest and most compact sources emitting at radio wavelengths in star forming regions. As an outcome, extremely rich populations of compact emitting sources, most of them a few hundreds of AU in size (when resolved), are unveiled in these regions

\footnotetext{
${ }^{1}$ Departamento de Astronomía, Universidad de Guanajuato, México.

${ }^{2}$ Instituto de Radioastronomía y Astrofísica, Universidad Nacional Autónoma de México, México.

${ }^{3}$ Mesoamerican Center for Theoretical Physics, Universidad Autónoma de Chiapas, México.

${ }^{4}$ Max Planck Institut für Radioastronomie, Germany.
}

(e.g., Forbrich et al. 2016; Medina et al. 2018). The nature of these Compact Radio Sources (CRS) is not unique, as shown by the variety of spectral indices that they exhibit, among other properties. Furthermore, special attention has been recently paid to the particular case of CRS associated with UCHII regions (i.e., very close to the massive star). Good examples can be found in W3 $(\mathrm{OH})$ (Kawamura \& Masson 1998; Dzib et al. 2013a) and NGC6334A (Carral et al. 2002; Rodríguez et al. 2014). The detection of these sources, associated with the probably densest part of the cloud, is challenging because they are usually weak compared to the extended freefree emission of the UCHII region where they are possibly embedded. In a recent systematic study 
(Masqué et al. 2017, hereafter MRT2017), 12 UCHII regions were observed to obtain an unbiased census of CRS associated with them. They reported 13 CRS, showing that the above-mentioned association is common. The significant number of CRS found allowed MRT2017 to classify them into two main types depending on the position of the compact sources with respect to the peak emission of the UCHII region. The CRS that appear projected close to the peak (i.e., possibly embedded in the dense ionized gas of the region) were called Type I sources, whereas the CRS scattered around the UCHII region were called Type II sources. The authors argued that Type I sources probably correspond to photoevaporating objects with high mass depletion rates as a consequence of the harsh irradiation from the nearby massive star. The large amount of expanding plasma emanating from these objects, either associated or not with the exciting star, maintains large emission measures in a compact volume around it, and an UCHII region is observed (lasting $\approx 10^{5} \mathrm{yr}$ according to Hollenbach et al. 1994). On the other hand, Type II sources could be photo-evaporating objects with lower depletion rates because the massive star is not so nearby. Their low mass depletion rates would prevent producing an observable UCHII region around them, and the CRS appears 'naked'. As they are probably long-lived, Type II objects are expected to be on average more evolved than Type I objects, and some of them could be pre-main sequence stars.

In this paper we present a follow-up study of MRT2017 to explore further the nature of the CRS. The interpretation of the nature of CRS given in MRT2017 was tentative, but sufficient to provide a possible schematic evolution and elucidate the role of CRS in star forming regions. We will constrain observational properties of the CRS, particularly the spectral index, supported by new Q-band observations of 4 UCHII regions containing both types of CRS. The results presented here are important to probe the scheme proposed in MRT2107 and shed light on the evolution of young objects embedded in giant clouds. The $7 \mathrm{~mm}$ observations are described in $\S 2$. In $\S 3$ we give our observational results and their discussion is presented in $\S 4$. Finally, in $\S 5$ we list our conclusions.

\section{OBSERVATIONS AND DATA REDUCTION}

\subsection{Source Selection}

We observed the UCHII regions G28.29-0.36, G35.20-1.74, G60.88-0.13 and G61.48+0.09A. All these regions are excited by late $\mathrm{O}$ or early $\mathrm{B}$ type stars and are part of the original selection of MRT2017. These authors found that G28.29-0.36 and G35.20-1.74 contain CRS of Type I, whereas G60.88-0.13 and G61.48+0.09A have Type II CRS. Our UCHII regions are located at fairly high declinations, since at $7 \mathrm{~mm}$ the quality of the observations strongly depends on source elevation. At the same time, we excluded the most compact UCHII regions because the CRS can be difficult to isolate in their corresponding maps.

G28.29-0.36, located at $3.1 \mathrm{kpc}$, is associated with IRAS 18416-0420. The maps of Kurtz et al. (1994) show that this UCHII region is composed of two peaks aligned north-south, with the CRS associated with the northern one. The G35.20-1.74 UCHII region is part of the W48 complex (Zeilik \& Lada 1978) located at $3.3 \mathrm{kpc}$ (Zhang et al. 2009). The $\mathrm{cm}$ emission at subarcsec scales shows a cometary shape with the tip oriented to the NE (Wood \& Churchwell 1989; Kurtz et al. 1994). The CRS is found less than $1^{\prime \prime}$ away from the peak of the UCHII region and very close to its geometric center. The G60.88-0.13 region, or Sh 87, is located at $2.1 \mathrm{kpc}$. Several IR sources were found in the field (Campbell et al. 1989), suggesting that a cluster of YSO surrounding the UCHII region is present. Within uncertainties, the CRS is coincident with one of these IR sources. Finally, G61.48+0.09A is embedded in the emission nebula Sh $2-88 \mathrm{~B}$, for which we adopted a distance of $2.0 \mathrm{kpc}$ (Crampton et al. 1978). This UCHII region shows compact northern emission and some southern extended emission. We found two CRSs embedded in the southern part, which is filtered out by our observations, and we consider that these CRS are detached from the densest part of the UCHII region. One of them is resolved, and has an arc-shaped morphology.

\section{2. $7 \mathrm{~mm}$ Continuum Observations and Mapping Procedures}

On 2016 May 22 and 24, we observed four UCHII regions of the sample of 12 regions observed at $1.3 \mathrm{~cm}$ by MRT2017 (program VLA/16A-003). The observations were performed in the $7 \mathrm{~mm}$ band with the VLA in the B configuration. We tuned the frequency range of 40.0-47.9 GHz arranged in 64 spectral windows of 64 channels each. Each channel had a width of $2 \mathrm{MHz}$. We used the 3 bit samplers with full polarization and 3 seconds as the integration time. The flux and bandpass calibrator was 3C286. Pointing corrections were applied for telescope slews larger 
TABLE 1

OBSERVED UCHII REGIONS AND SUMMARY OF THE VLA OBSERVATIONS

\begin{tabular}{|c|c|c|c|c|c|c|c|}
\hline \multirow{2}{*}{$\begin{array}{l}\text { UCHII } \\
\text { name }\end{array}$} & \multirow{2}{*}{$\begin{array}{l}\text { Distance }^{\mathrm{a}} \\
\quad(\mathrm{kpc})\end{array}$} & \multicolumn{2}{|c|}{ Pointing Center } & \multirow{2}{*}{$\begin{array}{c}\text { Gain } \\
\text { Calibrator }\end{array}$} & \multirow{2}{*}{$\begin{array}{c}\text { Positional Accuracy }{ }^{\mathrm{b}} \\
\text { (mas) }\end{array}$} & \multirow{2}{*}{$\begin{array}{c}S_{\nu}^{\mathrm{cal}, \mathrm{c}} \\
(\mathrm{Jy})\end{array}$} & \multirow{2}{*}{$\begin{array}{l}\text { Spectral } \\
\text { Index }^{\mathrm{d}}\end{array}$} \\
\hline & & $\alpha(\mathrm{J} 2000)$ & $\delta(\mathrm{J} 2000)$ & & & & \\
\hline G28.29-0.36 & 3.1 & $18^{\mathrm{h}} 44^{\mathrm{m}} 15^{\mathrm{s}} 097$ & $-04^{\circ} 17^{\prime} 55^{\prime \prime} 29$ & J1851-0035 & 150 & 0.68 & -0.54 \\
\hline G35.20-1.74 & 3.3 & $19^{\mathrm{h}} 01^{\mathrm{m}} 46^{\mathrm{s}} 490$ & $01^{\circ} 13^{\prime} 24^{\prime \prime} 65$ & J1851-0035 & 150 & 0.68 & -0.54 \\
\hline G60.88+0.13 & 2.1 & $19^{\mathrm{h}} 46^{\mathrm{m}} 20^{\mathrm{s}} 130$ & $24^{\circ} 35^{\prime} 29^{\prime \prime} 39$ & $\mathrm{~J} 1931+2243$ & 2 & 0.36 & -0.41 \\
\hline G61.48+0.09 & 2.0 & $19^{\mathrm{h}} 46^{\mathrm{m}} 49^{\mathrm{s}} 202$ & $25^{\circ} 12^{\prime} 48^{\prime \prime} 05$ & $\mathrm{~J} 1931+2243$ & 2 & 0.36 & -0.41 \\
\hline
\end{tabular}

${ }^{\text {a }}$ See references in the text.

${ }^{\mathrm{b}}$ Obtained from the NRAO database.

${ }^{\mathrm{c}}$ Bootstrapped flux density of gain calibrator at $41.128 \mathrm{GHz}$.

${ }^{\mathrm{d}}$ Spectral index of gain calibrator derived from the outcome of CASA tasks.

TABLE 2

PARAMETERS OF THE VLA MAPS

\begin{tabular}{|c|c|c|c|c|c|}
\hline \multirow[b]{2}{*}{ UCHII name } & \multicolumn{2}{|c|}{ Maps with all the visibilities ${ }^{\mathrm{a}}$} & \multicolumn{2}{|c|}{ Maps without short spacings ${ }^{b}$} & \multirow[b]{2}{*}{$N_{\text {sources }}^{\mathrm{c}}$} \\
\hline & $\begin{array}{l}\text { Beamsize } \\
\left({ }^{\prime \prime} \times^{\prime \prime} ;{ }^{\circ}\right)\end{array}$ & $\begin{array}{c}\text { map } r m s \text { noise } \\
\left(\mu \mathrm{Jy} \mathrm{bm}{ }^{-1}\right)\end{array}$ & $\begin{array}{l}\text { Beamsize } \\
\left({ }^{\prime \prime} x^{\prime \prime} ;{ }^{\circ}\right)\end{array}$ & $\begin{array}{c}\text { map } r m s \text { noise } \\
\left(\mu \mathrm{Jy} \mathrm{bm}{ }^{-1}\right)\end{array}$ & \\
\hline G28.29-0.36 & $0.160 \times 0.119 ;-19.2$ & 120 & $0.138 \times 0.099 ;-19.7$ & 70 & 1 \\
\hline G35.20-1.74 & $0.148 \times 0.124 ;-33.2$ & 300 & $0.127 \times 0.106 ;-35.2$ & 90 & 2 \\
\hline $\mathrm{G} 60.88+0.13$ & $0.130 \times 0.116 ;-84.3$ & 45 & $0.113 \times 0.100 ; 89.4$ & 60 & 1 \\
\hline $\mathrm{G} 61.48+0.09$ & $0.130 \times 0.117 ;-89.4$ & 80 & $0.113 \times 0.100 ;-89.7$ & 50 & 3 \\
\hline
\end{tabular}

${ }^{\mathrm{a}}$ Maps weighted with robust 0 .

${ }^{\mathrm{b}}$ Maps constructed with uniform weighting. We removed structures larger than 0.5 (see text).

${ }^{\mathrm{c}}$ Number of CRS associated with the region. We included the newly discovered sources.

than 10 degrees on the sky. We employed integrations of 1 to 1.5 minutes on-source, preceded and followed by integrations of 25 to $40 \mathrm{sec}$ on the gain calibrator to obtain a total on-source time of about 6 minutes. This sequence permits calibration solutions in intervals below the typical time scale of atmospheric phase fluctuations (which can be highly variable at the high frequency bands of the VLA) due to turbulence in the troposphere. The observational parameters are listed in Table 1.

The data were calibrated with the Common Astronomical Software Applications (CASA) package through the pipeline provided by NRAO. The CLEAN task of CASA with the nterms parameter set to 2 was used to construct the maps. We obtained a first set of maps setting the robust parameter to 0 to search for all the emission components. In order to remove extended emission from the maps, we re-imaged the fields using only uv-distances larger than $515 \mathrm{k} \lambda$, equivalent to mapping only structures smaller than 0.55 in size, and with uniform weighting. This cut was chosen to employ the same uv-range as that used in the synthesis of the $1.3 \mathrm{~cm}$ maps of MRT2017. This is a requirement for a proper com- parison between the maps at both wavelengths in the forthcoming analysis. The parameters of the $7 \mathrm{~mm}$ maps are shown in Table 2.

\section{RESULTS}

In Figure 1 we show our VLA $7 \mathrm{~mm}$ maps. The color scale corresponds to the maps obtained including all the visibilities; the contours show only the longer baseline data. The G28.29-0.36 and G35.20-1.74 regions are more extended than the nominal largest angular scale structure visible to the array (4", observing at the $7 \mathrm{~mm}$ band in the B configuration); hence, we only recover the brightest emission of these regions. On the other hand, the maps without extended structures shown as contours appear cleaner and the compact structures of the regions are clearly seen. In these maps, all the CRS reported in MRT2017 for these four UCHII regions are detected at $7 \mathrm{~mm}$, confirming that they are real structures and not artifacts of the maps. Using the nomenclature of MRT2017, they are G28-VLA1, G35-VLA1, G60-VLA1, G61-VLA1 and G61-VLA2; they are shown in Figure 2.

We found two additional CRS not reported in MRT2017 (we name them G35-VLA2 and 

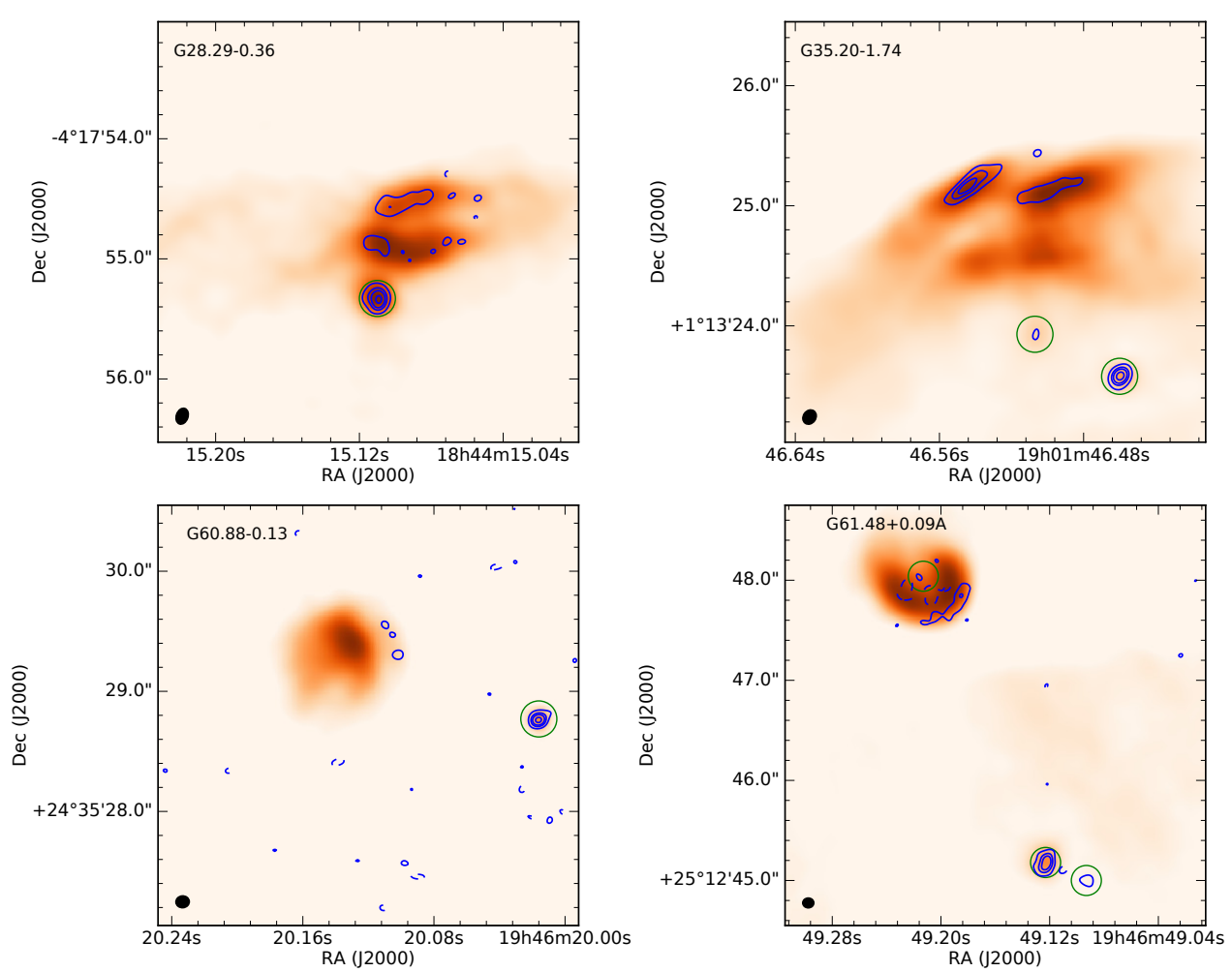

Fig. 1. Continuum $7 \mathrm{~mm}$ emission maps of the observed regions listed in Table 1 constructed with all the visibilities (color scale) with the maps constructed with baselines longer than $515 \mathrm{k} \lambda$ superimposed (contours). For the contours, levels are $-3,3,7,10$, and 15 times the rms noise level shown in Column 6 of Table 2. The color-scale maps are smoothed to an angular resolution of $0.2^{\prime \prime}$ to improve the appearance. The green circles indicate the location of the CRS reported in Table 3. The synthesized beam of the maps without short spacings is shown in the bottom right corner. The color figure can be viewed online.

TABLE 3

OBSERVED FLUXES AND SPECTRAL INDICES FOR THE CRSS

\begin{tabular}{|c|c|c|c|c|c|c|c|c|}
\hline \multirow[b]{2}{*}{ Source } & \multicolumn{2}{|c|}{ Coordinates $^{\mathrm{a}}$} & \multirow{2}{*}{$\begin{array}{l}S_{7 \mathrm{~mm}} \\
(\mathrm{mJy})\end{array}$} & \multirow{2}{*}{$\begin{array}{c}I_{7 \mathrm{~mm}}^{\text {Peak }} \\
\left(\mathrm{mJy} \mathrm{bm}^{-1}\right)\end{array}$} & \multirow{2}{*}{$\begin{array}{c}S_{1.3 \mathrm{~cm}}{ }^{\mathrm{b}} \\
(\mathrm{mJy})\end{array}$} & \multirow{2}{*}{$\begin{array}{c}I_{1.3 \mathrm{~cm}}^{\text {Peak }} \\
\left(\mathrm{mJy} \mathrm{bm}^{-1}\right)\end{array}$} & \multirow[b]{2}{*}{$\alpha_{S}^{\mathrm{c}}$} & \multirow[b]{2}{*}{$\alpha_{I}^{\mathrm{d}}$} \\
\hline & $\alpha(\mathrm{J} 2000)$ & $\delta(\mathrm{J} 2000)$ & & & & & & \\
\hline G28-VLA1 & $18: 44: 15.110$ & -4.17 .55 .33 & $3.0 \pm 0.2$ & $1.79 \pm 0.08$ & $3.41 \pm 0.22$ & $1.79 \pm 0.08$ & $-0.18 \pm 0.13$ & $0.00 \pm 0.09$ \\
\hline G35-VLA1 & 19:01:46.460 & 01.13 .23 .58 & $5.7 \pm 0.2$ & $4.8 \pm 0.1$ & $5.88 \pm 0.23$ & $4.53 \pm 0.11$ & $-0.05 \pm 0.07$ & $0.09 \pm 0.05$ \\
\hline G35-VLA2 & 19:01:46.507 & 01.13 .23 .93 & $1.0 \pm 0.4$ & $1.09 \pm 0.21$ & $1.0 \pm 0.3$ & $0.89 \pm 0.16$ & $-0.1 \pm 0.7$ & $0.3 \pm 0.4$ \\
\hline G60-VLA1 & $19: 46: 20.016$ & 24.35 .28 .77 & $1.01 \pm 0.07$ & $1.04 \pm 0.04$ & $0.31 \pm 0.02$ & $0.34 \pm 0.01$ & $1.69 \pm 0.13$ & $1.62 \pm 0.07$ \\
\hline G61-VLA1 & 19:46:49.123 & 25.12 .45 .18 & $2.4 \pm 0.3$ & $1.1 \pm 0.1$ & $3.5 \pm 0.3$ & $1.39 \pm 0.09$ & $-0.56 \pm 0.21$ & $-0.28 \pm 0.15$ \\
\hline G61-VLA2 & 19:46:49.093 & 25.12 .45 .00 & $0.6 \pm 0.1$ & $0.54 \pm 0.06$ & $0.40 \pm 0.06$ & $0.35 \pm 0.03$ & $0.5 \pm 0.3$ & $0.61 \pm 0.20$ \\
\hline G61-VLA3 & $19: 46: 49.216$ & 25.12 .48 .03 & $0.28 \pm 0.07$ & $0.28 \pm 0.07$ & $0.73 \pm 0.16$ & $0.56 \pm 0.08$ & $-1.4 \pm 0.5$ & $-1.0 \pm 0.4$ \\
\hline
\end{tabular}

a Typical positional uncertainties are $\approx 150$ mas for the CRSs associated with G28.29-0.36 and G35.20-1.74 (due to the poor positional accuracy of the J1851-0035 calibrator); and $\leq 30$ mas for the CRSs associated with G60.88+0.13 and G61.48+0.09.

${ }^{\mathrm{b}}$ Obtained from the $1.3 \mathrm{~cm}$ maps of Masqué et al. (2017) convolved to the $7 \mathrm{~mm}$ beam size of the maps shown in Figures 1 and 2 (contour maps).

${ }^{\mathrm{c}}$ Spectral index derived using integrated flux.

${ }^{\mathrm{d}}$ Spectral index derived using peak intensity.

G61-VLA3, and they are also shown in Figure 2). To confirm these new detections, we inspected the $1.3 \mathrm{~cm}$ maps of the corresponding UCHII regions and found that these new sources were marginally detected in the MRT2017 maps. They both share the characteristic of being centered on the UCHII 
TABLE 4

PHYSICAL PARAMETERS OF THE MARGINALLY RESOLVED CRSS

\begin{tabular}{lccccc}
\hline & $\theta_{M} \times \theta_{m} ;$ P.A $^{\mathrm{a}}$ & Size & $T_{\mathrm{B}}^{\mathrm{b}}$ & & $E M^{\mathrm{b}}$ \\
Source & $\left(\right.$ mas $^{\mathrm{m}}$ mas $\left.^{\circ}{ }^{\circ}\right)$ & $(\mathrm{AU} \times \mathrm{AU})$ & $(\mathrm{K})$ & $\tau^{\mathrm{b}}$ & $\left(10^{8} \mathrm{~cm}^{-6} \mathrm{pc}^{\mathrm{b}}\right.$ \\
\hline G28-VLA1 & $114 \pm 15 \times 70 \pm 30 ; 48 \pm 20$ & $340 \pm 50 \times 200 \pm 80$ & $360 \pm 160$ & $0.036 \pm 0.016$ & $3.0 \pm 1.3$ \\
G35-VLA1 & $61 \pm 8 \times 37 \pm 9 ; 136 \pm 20$ & $200 \pm 30 \times 120 \pm 30$ & $2300 \pm 600$ & $0.26 \pm 0.08$ & $22 \pm 7$ \\
G61-VLA1 & $155 \pm 23 \times 60 \pm 30 ; 160 \pm 8$ & $310 \pm 50 \times 120 \pm 50$ & $240 \pm 120$ & - & - \\
\hline
\end{tabular}

${ }^{\text {a }}$ Size is deconvolved from the beam of each map (see Column 4 of Table 2).

${ }^{\mathrm{b}}$ Brightness temperature (fourth column), optical depth (fifth column) and emission measure (sixth column) obtained in the same fashion as MRT2107, but with the expressions adapted for the frequency of $43.94 \mathrm{GHz}$ (i.e. the central frequency of the observed $7 \mathrm{~mm}$ band), and assuming an electron temperature of $10^{4} \mathrm{~K}$.

region and they could be associated with the exciting source. The detection of additional CRSs agrees with the scenario of these sources being common in the surroundings of the UCHII regions, most of them being extremely weak and hard to detect.

As a comparison, the contours of the $1.3 \mathrm{~cm}$ continuum maps convolved to the larger $7 \mathrm{~mm}$ beam are also displayed in Figure 2. As a general trend, the compact sources are unresolved or marginally resolved at both wavelengths and, thus, they appear very similar in the maps. Only G35-VLA2 shows an offset of $\approx 0 . \prime 07$ between the two frequencies. This displacement is smaller than our estimated positional error and so it is unlikely to be real.

Table 3 shows the measured flux densities at $7 \mathrm{~mm}$ (and $1.3 \mathrm{~cm}$ using the MRT2017 data) and the spectral indices for the seven CRS. Flux densities and peak intensities were derived by fitting two dimensional Gaussians to each CRS in the same manner for both bands (with the $1.3 \mathrm{~cm}$ maps convolved to the $7 \mathrm{~mm}$ beam size). From these parameters we derived spectral indices for the integrated flux densities of the CRSs $\left(\alpha_{S}\right)$ and for their peak intensities $\left(\alpha_{I}\right)$. If the source is resolved, these two indices might differ if inhomogeneities in the source structure are present. Table 4 shows the deconvolved sizes (angular and linear) of the three CRSs that were resolved, at least marginally, in the fit. Despite the large errors, an assessment of the true physical size of the source can be obtained. Moreover, in Table 4 we show physical parameters derived from the flux density and size of the CRS, namely, brightness temperature, optical depth and emission measure. As can be seen in Table 4, G35-VLA1 appears to be smaller and denser than the other sources. These physical parameters are consistent with those derived in MRT2017. Thus, the $1.3 \mathrm{~cm}$ and $7 \mathrm{~mm}$ continuum emission arises from the same region, at least in the resolved sources.

\section{ANALYSIS OF SELECTED SOURCES}

In this section we analyze the nature of the $7 \mathrm{CRS}$ detected in our $7 \mathrm{~mm}$ observations. A close examination of the properties of these CRSs, listed in Tables 3 and 4, shows that their properties are varied, suggesting a different physical nature for these sources. Thus, in the following we analyze them individually:

G28-VLA1: The possibility that this source is a very small HCHII region was discarded by MRT2017 because they showed that G28-VLA1 is unlikely to host the massive star exciting the region. The remaining possibilities are externally photoevaporated disk/clump winds and thermal radio jets associated with young low-mass objects. Despite the different launching mechanism and structure of the outflowing gas, their observational signposts are often very similar. Unfortunately, our angular resolution yields a deconvolved size too uncertain for an accurate morphological inspection (either at $1.3 \mathrm{~cm}$ or $7 \mathrm{~mm}$ ). Furthermore, our observing frequency is well above the turn-over frequency $(\approx 10 \mathrm{GHz}$ for G28-VLA1) as indicated by the low values of the optical depth $\left(\approx 4 \times 10^{-2}\right)$. This results in spectral indices $\alpha_{I}(\approx 0)$ and $\alpha_{S}(\approx-0.2)$, consistent with optically thin thermal emission of ionized gas. Flat spectral indices are expected for jets and disk winds when observed at frequencies high enough for essentially the whole object to become optically thin (Reynolds 1986; Hollenbach et al. 1994; Anglada et al. 2018).

On the other hand, the emission measure of G28-VLA1 $\left(3 \times 10^{8} \mathrm{~cm}^{-2} \mathrm{pc}\right)$ is in excellent agreement with the typical values obtained by Hollenbach et al. (1994) for their photo-evaporating disk wind models, but is significantly higher than that expected for a jet (e.g, see Rodríguez et al. 1990; Curiel et al. 1993). Furthermore, jets usually show additional structures, such as knots resulting from previous ejecta (e.g. Martí et al. 1993), which are not seen in our maps. Therefore, we favor the possi- 

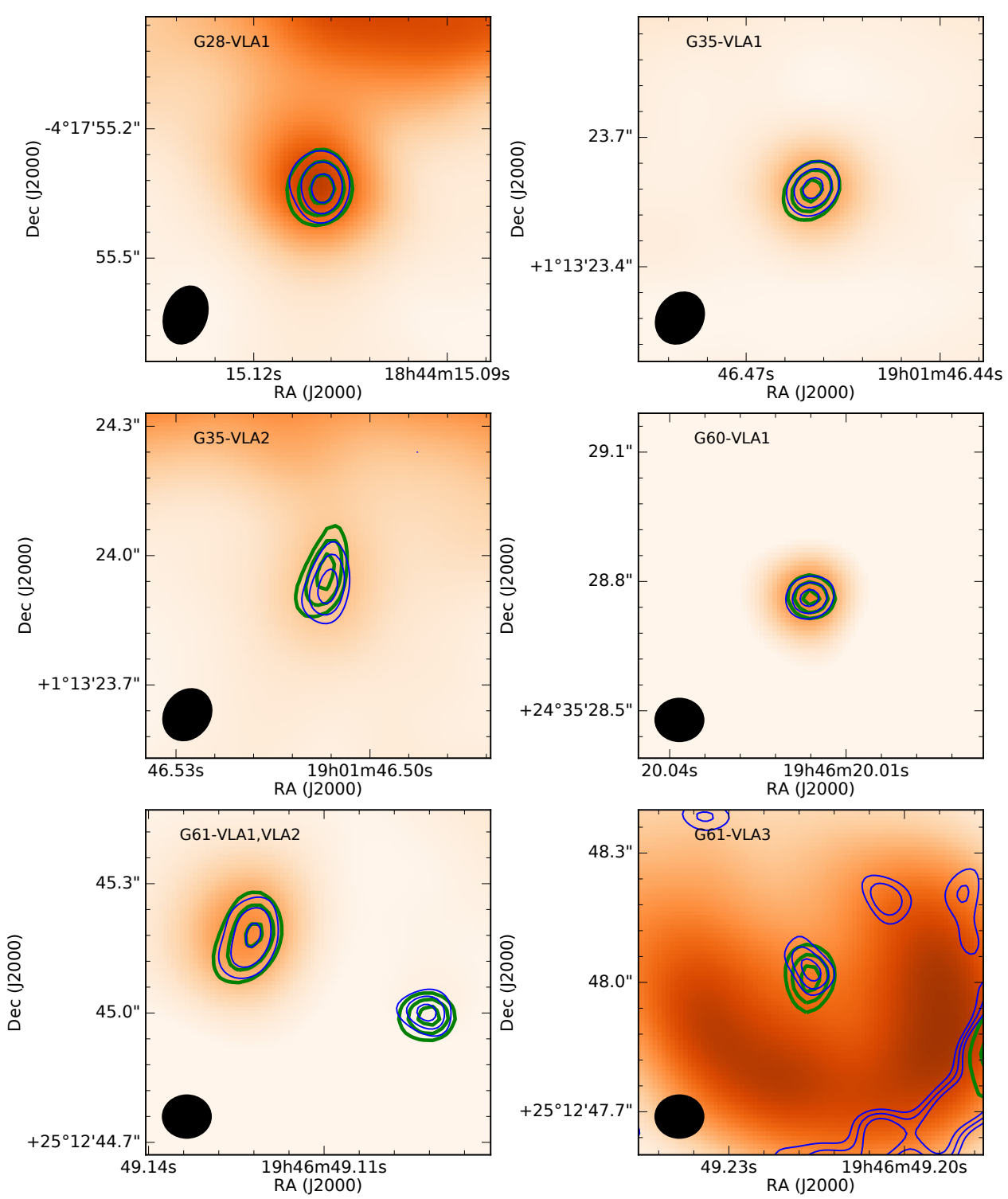

Fig. 2. Zooms on the CRS indicated by green circles in Figure 1. The color scale represents the 7 mm maps constructed with all the visibilities; the blue contours represent the levels corresponding to $50 \%, 70 \%$ and $90 \%$ of the brightness peak of the CRS, and the green contours represent the same, but for the $1.3 \mathrm{~cm}$ continuum emission. The maps shown with a color scale are the same as those shown in Figure 1 (i.e. smoothed to an angular resolution of $0.2^{\prime \prime}$ ) but with a modified range. Both maps shown with contours have been convolved to the same synthesized beam, which is shown in the bottom left corner. The color figure can be viewed online.

bility that the optically thin emission of G28-VLA1 is produced by a photo-evaporated disk/clump wind.

G35-VLA1: This CRS has the largest derived $E M\left(\approx 10^{9} \mathrm{~cm}^{-6} \mathrm{pc}\right)$ and a relatively compact size $(<100 \mathrm{AU})$ compared to other resolved CRSs (G28-VLA1 and G61-VLA1). Based upon size and density, MRT2017 proposed that G35-VLA1 is associated with the exciting star of the UCHII region, as its size is smaller than twice the gravitational radius. The large emission measure of G35-VLA1 rules out a jet as a possibility for this CRS. The turnover frequency for an emission measure of $2.2 \times 10^{9} \mathrm{~cm}^{-6} \mathrm{pc}$ is $\approx 25 \mathrm{GHz}$. At our observing frequency of $45 \mathrm{GHz}$ the gas should be completely transparent. This seems consistent with the flat values for the spectral indexes. However, within the uncertainties, we 
cannot discard a slight step in these indices, and the optical depth $(\approx 0.3)$ is too large to consider optically thin emission.

By comparing $\alpha_{S}$ and $\alpha_{I}$ of Table 3 , we see that $\alpha_{S}<\alpha_{I}$. The same result was found for some of the radio sources of Cepheus A by Garay et al. (1996). These authors conclude that a larger spectral index of the peak emission suggests the presence of an optically thick compact region at the center of the source. Considering G35-VLA1 as a trapped HCHII region, its optically thick part must be $<70 \mathrm{AU}$ in radius (see Table 5 of Keto 2003, where an O6 star is assumed, more massive than the star exciting G35.20-1.74). On the other hand, following the prescription of Hollenbach et al. (1994) for photoevaporating disk winds (see their equation 7.1 ) this radius varies between $36-168 \mathrm{AU}$, observing at $7 \mathrm{~mm}$ and for mass loss rates typical for Type I objects $\left(10^{-5}-10^{-6} M_{\odot} \mathrm{yr}^{-1}\right)$. These values are consistent with the deconvolved size of G35-VLA1 of Table 4. Because this size is well below our angular resolution, only a slight rise in $\alpha_{I}$ is observed. Therefore, the observational properties of G35-VLA1 suggest a density gradient in the CRS that could be the result of outflowing ionized material, or of a trapped HCHII region.

G35-VLA2: This CRS lies near the geometrical center of the G35.20-1.74 UCHII region and is clearly a Type I object according to MRT2017. The detection of G35-VLA2 is too marginal to reach any strong conclusion about its nature; additional observational constraints are required.

G60-VLA1: MRT2017 propose that this source is composed of ionized gas trapped in the potential well of a B-type star, forming a small HCHII region. This is consistent with the lower limit of EM of $7 \times 10^{7} \mathrm{~cm}^{-6} \mathrm{pc}$ for this CRS. Moreover, our derived spectral index is $\approx 1.7$, which indicates an optically thick gas, usually found at centimeter wavelengths in HCHII regions.

G61-VLA1: This CRS is the best-resolved of our sample and shows hints of an arc-like shape. The tip of the arc points towards G61-VLA2 suggesting that the latter is responsible for photo-evaporating the former. Both $\alpha_{S}$ and $\alpha_{I}$ for G61-VLA1 are clearly negative, showing unambiguously that the emission is mainly non-thermal. Other cases of negative spectral indices around proplyds have been previously reported (Mücke et al. 2002; Masqué et al. 2014), and are attributed to the presence of a relativistic electron population in a magnetized medium. Regardless of the mechanism that produces high-energy electrons, an estimation of the magnetic field can be obtained by assuming equipartition of energy between the magnetic field and relativistic particles. Following Pacholczyk (1970), the magnetic field estimated under these conditions is given by:

$$
B[\text { Gauss }]=\left[4.5(1+\chi) c_{12} L\right]^{2 / 7} R^{-6 / 7}
$$

where $L$ and $R$ are the radio luminosity and radius of the source (assuming spherical symmetry) given in cgs units, and $\chi$ is the energy ratio of the heavy particles to electrons. $L$ is obtained by integrating the area below the spectra derived from the $1.3 \mathrm{~cm}$ and $7 \mathrm{~mm}$ fluxes and extrapolated to the limits of 0.01 to $100 \mathrm{GHz}$ (e.g., Miley 1980). Thus, this parameter implicitly includes the dependence on the spectral index $\alpha$. The total luminosity in the radio domain is $1.6 \times 10^{30} \mathrm{erg} \mathrm{s}^{-1}$. The parameter $c_{12}$ is a function of the spectral index and frequency; tabulated values were given by Pacholczyk (1970). In our particular case its value is $2 \times 10^{7} \mathrm{Gauss}^{3 / 2} \mathrm{~s}$. For $R$ we calculated an equivalent source size $\theta_{\mathrm{eq}}=\left(\theta_{\mathrm{M}} \theta_{\mathrm{m}}\right)^{1 / 2}$ (see Column 2 of Table 4), which corresponds to a linear size of $R=1.4 \times 10^{15} \mathrm{~cm}$. The value of $\chi$ is very uncertain and varies from 1 to 2000 depending on the mixture of relativistic particles considered (e.g. electrons, positrons and/or protons). However, the exponent $2 / 7$ makes the magnetic field weakly dependent on $\chi$ and we do not expect changes beyond an order of magnitude. For this parameter, we adopted 40 as measured by Simpson et al. (1983) near the Earth.

We obtained a value of $B \approx 25 \mathrm{mG}$ which, despite being a rough number (it has about $50 \%$ uncertainty), it is well above other values found in a variety of radio sources showing thermal emission, such as Cepheus $\mathrm{A}(\approx 0.3 \mathrm{mG}$, Garay et al. 1996) or in proplyds like those found in the NGC 3603 region $(\approx 1 \mathrm{mG}$, Mücke et al. 2002). The high value of $B$ depends on the compact size of G61-VLA1 and suggests that the magnetic field increased its strength as a consequence of a prior contraction (either gravitational or driven by external pressure) suffered by these sources (Mouschovias \& Spitzer 1976). Consistently, the proplyds discussed in Mücke et al. (2002) are much larger than G61-VLA1 (10 ${ }^{4}$ vs. $\left.200 \mathrm{AU}\right)$. The tendency of $\alpha_{S}<\alpha_{I}$ can be interpreted as an increase of electron density in the central part of the source, similar to the G5.59-VLA1 proplyd (Masqué et al. 2014): given a mixture of thermal and nonthermal emission, the former will tend to dominate in the densest part of the source because it depends on $n_{e}^{2}$, where $n_{e}$ is the electron density, contrary to 
non-thermal emission, which depends on other parameters, like magnetic field or shock energetics.

Alternatively, a wind collision region (WCR) of two massive stars, where the most massive member is G61-VLA2, could explain the non-thermal nature of the emission and the morphology of G61-VLA1. Examples of WCR between two massive stars have been reported previously, such as in Cyg OB \#5 (Contreras et al. 1996, 1997; Dzib et al. 2013b). This scenario is favored by our value for the magnetic field, which matches well with that found in other WCR (20 mG in HD 93129A, del Palacio et al. 2016). The only caveat in this scenario is the lack of a point IR counterpart indicating the presence of a massive ionizing star in G61-VLA2, unless its IR emission is obscured by a small disk located edge on. A possible candidate is a 2MASS point source located $10^{\prime \prime}$ $(20,000 \mathrm{AU}$ at $2 \mathrm{kpc})$ to the west. With the present data, we cannot discriminate between a magnetized neutral object (i.e., a clump or protostellar disk) that is being photo-evaporated or a WCR between massive stars present in the region to explain the nature of G61-VLA1.

G61-VLA2: The spectral index of G61-VLA2 is similar to that of collimated winds (i.e., jets) or isotropic winds with a density gradient (0.6, Panagia \& Felli 1975). The lower limit of EM for this CRS $\left(4 \times 10^{7} \mathrm{~cm}^{-6} \mathrm{pc}\right)$ rules out the protostellar jet possibility, as they have lower expected values (Rodríguez et al. 1990; Curiel et al. 1993). On the other hand, an expanding ionized disk wind with a radial density gradient is an excellent match (in terms of spectral index) to the Hollenbach et al. (1994) model. Therefore, G61-VLA2 could be a photo-evaporating object, possibly a disk around a YSO. However, instead of having a photo-evaporated flow with a resolved volume (sizes > $200 \mathrm{AU}$ ) such as other CRS, the volume of the ionized flow of G61-VLA2 is so small that its optically thick region fills a significant fraction of the total volume. The nearby cometaryshaped G61-VLA1 supports the existence of a stellar/disk wind.

G61-VLA3: Despite its large uncertainty, the spectral index of G61-VLA3 is clearly negative $(\approx-1)$. This suggests gyro-synchrotron radiation produced in the magnetically-active corona of a young solar type star. This emission mechanism is commonly observed in star-forming regions containing low-mass YSO (e.g., Dzib et al. 2013b). Since they can show dramatic flux variations over days or hours, we obtained fluxes separately for the two observing days and compared their values to search for rapid flux variability. We observed differences of $40 \%$, which is slightly above the relative errors of our flux determination and consistent with a nonthermal nature of G61-VLA3. Nevertheless, future flux monitoring of this source is needed to assess flux variability. Also, we mapped the Stokes $V$ parameter to look for circular polarization at $1.3 \mathrm{~cm}$ and $7 \mathrm{~mm}$. The weak brightness of this CRS hinders a clear detection of polarized emission, but we obtained an upper limit of $10 \%$, from which we cannot discard the presence of circularly polarized emission and the possibility that G61-VLA2 is a magnetic star.

\section{DISCUSSION AND CONCLUSIONS}

We detected seven CRS located in four UCHII regions previously studied by MRT2017. The analysis of their properties yields that four of them have thermal and two non-thermal emission. One remaining source (G35-VLA2) is detected too marginally to elucidate its emission nature.

The four thermal sources have free-free emission arising from ionized gas in the CRS. According to the MRT2017 scenario, G28-VLA1 and G35-VLA1 are Type I sources and, hence, the ionized gas corresponds to a photo-evaporated flowing material with high mass depletion rates $\left(\dot{M}>10^{-6} M_{\odot} \mathrm{yr}^{-1}\right)$. On the other hand, G60-VLA1 and G61-VLA2 are Type II sources and have significantly lower, or even no, depletion rates. This scenario is in excellent agreement with our interpretation of the nature of G28-VLA1 as it appears well-centered on G28.29-0.36 and, at least partially, is feeding the UCHII region with its photo-evaporated outflowing material. The scenario is consistent with the isolated locations of CRSs G60-VLA1 and G61-VLA2, as they are unable to produce a sufficiently extended ionized region around them. It is worth noting that the possibility that G60-VLA1 is a trapped HII region is also consistent with the scenario discussed by Keto $(2002,2003)$, where instead of outflowing gas, the accretion proceeds through the HII region and the star is still growing in mass.

There is some ambiguity in the interpretation of the nature of G35-VLA1 as either being a trapped HII region or a photo-evaporated wind. Depending on each possibility, the material within the UCHII region could be expanding or infalling from/onto the CRS. Interestingly, these scenarios are not mutually exclusive if rotation is included (Keto \& Wood 2006; Keto 2007). In the resulting configuration, the gas is outflowing except for a given range of equatorial angles with respect to the rotation axis, where the gas is infalling. This possibility implies bipolar morphologies, which are not observed for G35-VLA1 be- 
cause its compact size prevents us from determining its morphology with these observations. Additional observations with higher angular resolution (i.e., the A configuration) to explore the kinematics of the G35.20 UCHII region (e.g., through recombination lines) are crucial to shed light on this last possibility.

The non-thermal CRSs possibly have natures different than the photo-evaporating objects. G61-VLA1 is a Type II source and, hence, consistent with the MRT2017 scenario. On the other hand, the location of G61-VLA3 centered on G61.48+0.09 and the nature of its radio emission mechanism are puzzling: it is difficult to explain the presence of a low mass YSO embedded in an UCHII region (i.e., Type I source). A possible scenario can be that G61-VLA3 is unrelated to the dense gas of the UCHII region but projected in its direction on the sky. Such an arrangement is unlikely, but not impossible (e.g. Trejo \& Rodríguez 2010; Dzib et al. 2016). Future investigations to constrain the nature of these CRS are required to conclusively confirm or reject the scenario proposed by MRT2017.

JMM acknowledges the support of DAIP-UG (CIIC2020 project 068/2020). L.L. acknowledges the support of DGAPA-UNAM (PAPIIT project IN112820) and CONACyT (Ciencia de frontera project 263356).

\section{REFERENCES}

Anglada, G., Rodríguez, L. F., \& Carrasco-González, C. 2018, A\&ARv, 26, 3

Campbell, B., Persson, S. E., \& Matthews, K. 1989, AJ, 98, 643

Carral, P., Kurtz, S. E., Rodríguez, L. F., et al. 2002, AJ, 123,2574

Contreras, M. E., Rodriguez, L. F., Gomez, Y., \& Velazquez, A. 1996, ApJ, 469, 329

Contreras, M. E., Rodriguez, L. F., Tapia, M., et al. 1997, ESASP 402, 'Hipparcos - Venice '97', 401

Crampton, D., Hutchings, J. B., \& Cowley, A. P. 1978, ApJ, 223, 79

Curiel, S., Rodriguez, L. F., Moran, J. M., \& Canto, J. 1993, ApJ, 415, 191

del Palacio, S., Bosch-Ramon, V., Romero, G. E., \& Be- naglia, P. 2016, A\&A, 591, 139

Dzib, S. A., Ortiz-León, G. N., Loinard, L., et al. 2016, ApJ, 826, 201

Dzib, S. A., Rodríguez, L. F., Loinard, L., et al. 2013a, ApJ, 763, 139

Dzib, S. A., Rodríguez-Garza, C. B., Rodríguez, L. F., et al. 2013b, ApJ, 772, 151

Forbrich, J., Rivilla, V. M., Menten, K. M., et al. 2016, ApJ, 822, 93

Garay, G., Ramírez, S., Rodríguez, L. F., Curiel, S., \& Torrelles, J. M. 1996, ApJ, 459, 193

Hollenbach, D., Johnstone, D., Lizano, S., \& Shu, F. 1994, ApJ, 428, 654

Kawamura, J. H. \& Masson, C. R. 1998, ApJ, 509, 270

Keto, E. 2002, ApJ, 580, 980

2003, ApJ, 599, 1196

2007, ApJ, 666, 976

Keto, E. \& Wood, K. 2006, ApJ, 637, 850

Kurtz, S., Churchwell, E., \& Wood, D. O. S. 1994, ApJS, 91, 659

Martí, J., Rodríguez, L. F., \& Reipurth, B. 1993, ApJ, 416, 208

Masqué, J. M., Dzib, S., \& Rodríguez, L. F. 2014, ApJ, 797,60

Masqué, J. M., Rodríguez, L. F., Trinidad, M. A., et al. 2017, ApJ, 836, 96

Medina, S.-N. X., Dzib, S. A., Tapia, M., Rodríguez, L. F., \& Loinard, L. 2018, A\&A, 610, 27

Miley, G. 1980, ARA\&A, 18, 165

Mouschovias, T. Ch. \& Spitzer, L. Jr. 1976, ApJ, 210, 326

Mücke, A., Koribalski, B. S., Moffat, A. F. J., Corcoran, M. F., \& Stevens, I. R. 2002, ApJ, 571, 366

Pacholczyk, A. G. 1970, Radio astrophysics. Nonthermal processes in galactic and extragalactic sources (San Francisco, CA: Freeman)

Panagia, N. \& Felli, M. 1975, A\&A, 39, 1

Reynolds, S. P. 1986, ApJ, 304, 713

Rodríguez, L. F., Ho, P. T. P., Torrelles, J. M., Curiel, S., \& Canto, J. 1990, ApJ, 352, 645

Rodríguez, L. F., Masqué, J. M., Dzib, S. A., Loinard, L., \& Kurtz, S. E. 2014, RMxAA, 50, 3

Simpson, J. A., Wefel, J. P., \& Zamow, R. 1983, ICRC, 10,322

Trejo, A. \& Rodríguez, L. F. 2010, RMxAA, 46, 357

Wood, D. O. S. \& Churchwell, E. 1989, ApJ, 340, 265

Zeilik, M. I. \& Lada, C. J. 1978, ApJ, 222, 896

Zhang, Q., Wang, Y., Pillai, T., \& Rathborne, J. 2009, ApJ, 696, 268

Josep M. Masqué, Miguel A. Trinidad, and Carlos A. Rodríguez-Rico: Departamento de Astronomía, Universidad de Guanajuato, Apdo. Postal 144, 36000 Guanajuato, México.

Luis F. Rodríguez, Laurent Loinard, and Stan Kurtz: Instituto de Radioastronomía y Astrofísica, Universidad Nacional Autónoma de México, Morelia 58089, México.

Luis F. Rodríguez: Mesoamerican Center for Theoretical Physics, Universidad Autónoma de Chiapas, Carretera Emiliano Zapata Km. 4, Real del Bosque (Terán), 29050 Tuxtla Gutiérrez, Chiapas, México.

Sergio A. Dzib and Sac N. Medina: Max Planck Institut für Radioastronomie, Auf dem Hügel 69, D-53121 Bonn, Germany. 